\title{
A Modification of the Random Curds Model by Markov Chain as a Design Tool in Architecture
}

\author{
Ljiljana PETRUŠEVSKI, Ivana ĆIROVIĆ
}

\begin{abstract}
The procedure of generating fractal rhythm in architecture with the help of the random curds model proposed by Carl Bovill had its limitations in practice, related to the number of possible states of architectural elements. Namely, the binary relation: "survives" - "does not survive" between the model units was directly transferred to relationships of architectural elements, which, consequently, can be only in one of these two possible conditions: "this one exists - that one exists". Authors of this paper, in their effort to overcome the aforementioned limitation, in addition to the cell that does not exist, suggest the existence of not only one, but of several different "living cells". The suggested procedure is interpreted as a Markov chain with several states, through several scales. This way, the authors created a modification of the random curds model in this paper, within which they achieved an arbitrary predefined, finite or countable infinite set of states.
\end{abstract}

Keywords: fractal rhythm in architecture; Markov chains; Random Curds; random fractals

\section{INTRODUCTION}

Elements, units or motifs of architectural and urban compositions identical or similar by their features, or by their role in the composition, cause the experience of visual rhythm in the observer with their multiple occurrence in the same, or modified form $[1,2,3]$.

Bovill $[4,5]$ proposed and described a method for defining rhythm in architecture with the help of random curds. The author defined rhythm determined in such a way as fractal rhythm. Random curds are objects of fractal geometry from the class of random fractals, described by Bovill [4, p.92] as 'disconnected set of points that has a clustered characteristic', and a set of unconnected points was named fractal dust, as suggested by Mandelbrot [6] who has given the name curdling to the procedure that produces a random fractal dust.

Bovill observed random curds as mathematical models of natural rhythm or natural distribution of elements, and he described the procedure of transferring that rhythm from a model to rhythm in architecture. The author's intention was to achieve variability and random clustering in architectural and urban compositions, similar to the one found in nature. Describing the procedure of transferring rhythm from a fractal model to rhythm in architecture, Bovill stated Park de la Villette as an illustration of the possibility to generate rhythm this way, where, instead of using the Euclid's mesh, units could have been distributed as random fractal dust, whose 'mix of order and disorder', according to the author [4, p.176], call into question the existing concept of order, i.e. its interpretation. Artists and scientists both ancient and modern have searched for common ideas that span areas as diverse as art, architecture, chemistry, physics and biology, with mathematics and geometry as a common language and found resonances at astronomical, human and sub-microscopic scales. They discovered the geometric bridge between architecture and patterns present in nature [e.g. 7, 8, 9]. Analyzing the potential role of geometry in architecture, Evans [10] notes that in the design process, geometry serves as a support to architects, as a scientific truth tested and proved in the context of another discipline - mathematics, where architects require and expect 'firmness and stability' from geometry $[10$, p.XXVII]. Another author, Ostwald [11, 12] expands the stated role of geometry as the scientific support for architects to the scientific authority, emphasizing that architects seldom accept geometry covertly, moreover, that they clearly underline the presence of the geometry system in an effort to provide their project with a source of authority, or to provide justification for their design decisions. The same author [13] also points out the existence of some sort of architects' faith in geometry to give sense to a building, where the significance of a geometric shape, or the sense attributed to it does not lie in its inherent characteristic as such, but in its assumed similarity and association with some ideal or original forms, or in a perceived similarity with natural forms.

In this paper, where we described the application of fractal geometry in determining fractal rhythm, the presumed relationship between geometry and a sense it introduces to architecture can be traced in two directions. The first one identifies fractal geometry with the geometry of nature, as it was characterized and described by its creator at its very birth: 'I conceived and developed a new geometry of nature' $[6, p .1]$. The second one, according to Ostwald [12], associates fractal geometry with a group of various scientific disciplines, 'whose only common denominator is the search for deterministic chaos within natural systems' [12, p.56], grouped under the same title of Complexity Science, and relying mostly on fractal geometry, e.g. chaos theory and non-linear dynamics. According to the same author [14], fractal geometry is less important per se for architects today, and more because they believe it can provide them a connection with nature, and with global change in science, as we have witnessed in the last few decades. The aforementioned global change in science is described by Prigogine [15] in the book of the same name as: 'the end of certainty', where he notices that we are now witnesses of abandoning 'one of the basic traditions of Western thought, the belief in certainty' $[15, \mathrm{p} .4]$. According to him [15], unlike classical science which emphasized order and stability and in which 'laws of nature express certitudes', now 'the meaning of the laws of nature 
changes radically, for they now express possibilities or probabilities' $[15, \mathrm{p} .4]$. And it should also be noted that Mandelbrot [6], at the very beginning of his book underlines that 'the most useful fractals', useful in terms of describing natural phenomena with them, are those that 'involve chance and both their regularities and their irregularities are statistical' [6, p.1]. And later, introducing random fractals in his essay, he says: 'The theory of probability is the only mathematical tool available to help map the unknown and the uncontrollable' [6, p.201]

Some authors $[16,17,18]$ have examined the possibilities of arranging elements in architectural and urban compositions with the help of random fractals, by introducing a component of chance, while simultaneously avoiding arbitrariness. That way fractal geometry as a "truth" verified and proved within another discipline (mathematics) can appear in architecture in a supporting role, but also as a possible source of scientific authority. Moreover, due to the described connections of fractal geometry and nature on the one hand, and the "new science" on the other, and in the role of a potential bringer of some kind of "sense", or meaning to architecture, if we assume that the perceived characteristics of randomness and "naturalness" in compositions generated with random fractals, will point us to the stated relations.

\section{RANDOM CURDS MODEL}

Random fractals, same as deterministic fractals, occur as a result of recursive construction procedures or algorithms, except that there is a component of chance in the process of generating random fractals. This way, in random fractals, which include random curds, selfsimilarity or invariance of form through several scales is statistic, in other words, it is present, but it is not deterministically defined. It is statistical self-similarity, and that feature, where viewing the larger part is insufficient to accurately predict the appearance of the smaller magnified part, is precisely the basic characteristic of natural fractals [19]. Moreover, studies have shown $[20,21]$ that the component of randomness is responsible for the visual feature of naturalness or natural-looking, as well as that the attribute of naturalness is a significant correlate of major preferences in experiential studies on aesthetic preferences [22].

According to Mandelbrot [6], random curds can be obtained by conducting a simple process of curdling. According to his explanation, it is a cascade process that results in contraction, and originates from an effort to 'mimic reality by purely geometric means' [6:84]. The author states clusters of galaxies and stars as an example of uneven distribution of matter in nature.

To construct these fractals, according to Mandelbrot, we need: 'a grid or lattice, made of intervals, squares, or cubes, each divided into $b^{\mathrm{E}}$ subintervals, subsquares, or subcubes; $b$ is the lattice base' [6:210], where $E$ is 1,2 or 3 , for line segments, squares or cubes, respectively. Curdling or random clustering is achieved by a series of random binary choices (using dice or coin) on the fate of each of $b^{E}$ subline segment, subsquare or subcube. With probability $p<1$, according to the author: 'the subinterval survives as a part of a precurd; otherwise, it dies off [6:211]. With the "surviving" intervals, squares, or cubes, we pass to the next step of the cascade process. Selecting the probability of event for each step of the process, as well as the number of steps, we can get a model of random clustering of elements with desired characteristics.

\subsection{A Reinterpretation of the Random Curds Model}

Following the terminology of cellular automata [23], we will call the described line segments, squares and cubes by a common name - cells. The initial state is a single living cell covered by the initial grid network which divides the living cell to new, smaller-scale cells. The probability of survival of each cell is $p$, and the probability of non-survival is $1-p$. The procedure is then repeated through several scales. In every next step (iteration), each of the living cells is divided to new scaled cells with the scaled grid network from the previous step, and the probability of survival of each of them is $p$, whereas the probability of non-survival is $1-p$. Probabilities of survival are variable, which means they are the same within one step, while they have different values through different steps, i.e. the probability of survival in an n-step is $p=p(n)$. The surviving cells from each step will be called living cells in further text, and non-surviving cells will be dead cells.

\subsection{Limitations of the Random Curds Model}

What is the result of implementing the algorithm after $n$ steps? A class of random curd fractal consists of living cells after the last step, and dead cells are visible in several scales in the grid, i.e. dead cells obtained through all previous steps, because their division is not performed in the subsequent steps (Fig. 1a). This way, in addition to the random curds fractal consisting of living cells (Fig. 1b), we also have interesting fractal rhythm provided by the present dead cells in several scales (Fig. 1c). Fig. 2 shows different presentations of that complementary fractal which additionally increases the potential of random curds fractals as a mathematical model of natural rhythm in architectural and urban compositions. In Fig. 2, white, unpainted cells represent the random curds fractal from Fig. 1.

As an example of implementation of the random curds model in architectural compositions, Fig. 3 shows a façade glass wall with square panels of different size and different level of transparency, generated through three iterations. The possibility of survival in the first iteration was $p=0.7$, in the second $p=0.7$, and in the third $p=0.6$.

In the upper row of images, living cells from the used mathematical model are treated as transparent and displayed with a transparency of $80 \%$, whereas dead cells are treated as non-transparent and displayed with a transparency of $15 \%$. So, it is quite clear we only have two states here, without the existence of some kind of transitional or intermediate states, which is particularly obvious in situations such as this, when two states are actually two opposite, or two final states: exists - does not exist; yes - no, or in this case: transparent - nontransparent. However, the authors would like to note that in nature, situations where phenomena occur in more states than just these two are more common, in other 
words, that opposite or very distant values are usually balanced by the existence of intermediate values. Therefore, in an effort to bring their generated rhythms in architecture as close to the appearance of the natural environment as possible, the authors of this paper generated the fractal structure shown in the second row of Fig. 3, with two possible states of living cells (transparency $40 \%$ and $80 \%$ ). The probabilities of survival $p$ have remained the same in the sum of both living states, in comparison to the previous example, except that the probability of each of the two possible states of living cells is $p / 2$, regardless of the state of the living cell from which it originates, and the probability of the cell's non-survival is $1-p$. Encouraged by the results, the authors begin to think about Markov chains with several states (through several scales), in whose transition probabilities they would incorporate the basic idea behind the random curds model - that not all cells survive, and that those that did not survive cannot produce new living cells in subsequent steps.

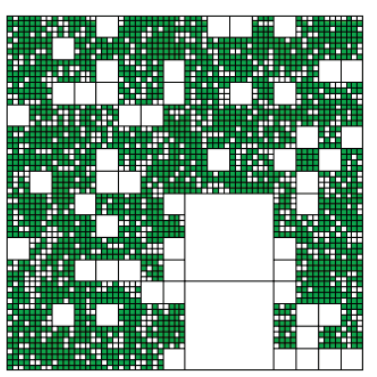

a)

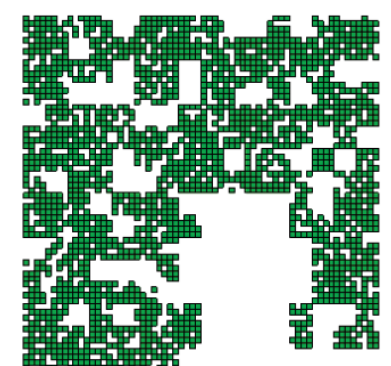

b)

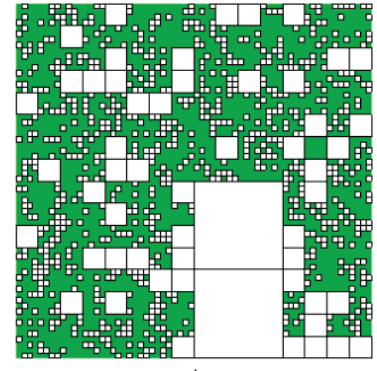

c)

Figure $1 \mathrm{a})$ Procedure of generating Random Curds class of fractals,

b) A Random Curds class fractal (living cells), c) Accentuated fractal rhythm of dead cells

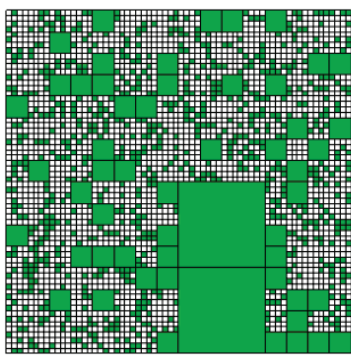

a)

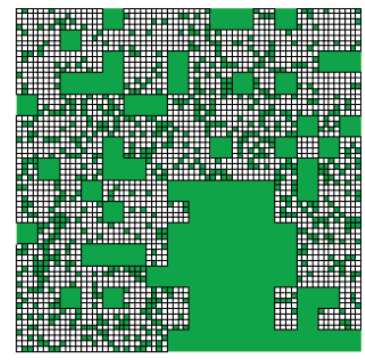

b)

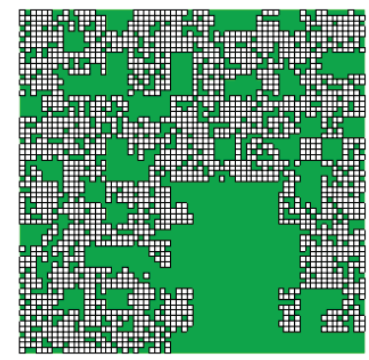

c)

Figure 2a) Individual presentation of dead cells through 3 iterations

b) Clustered dead cells from the first two iterations, c) Clustered dead cells from all three iterations
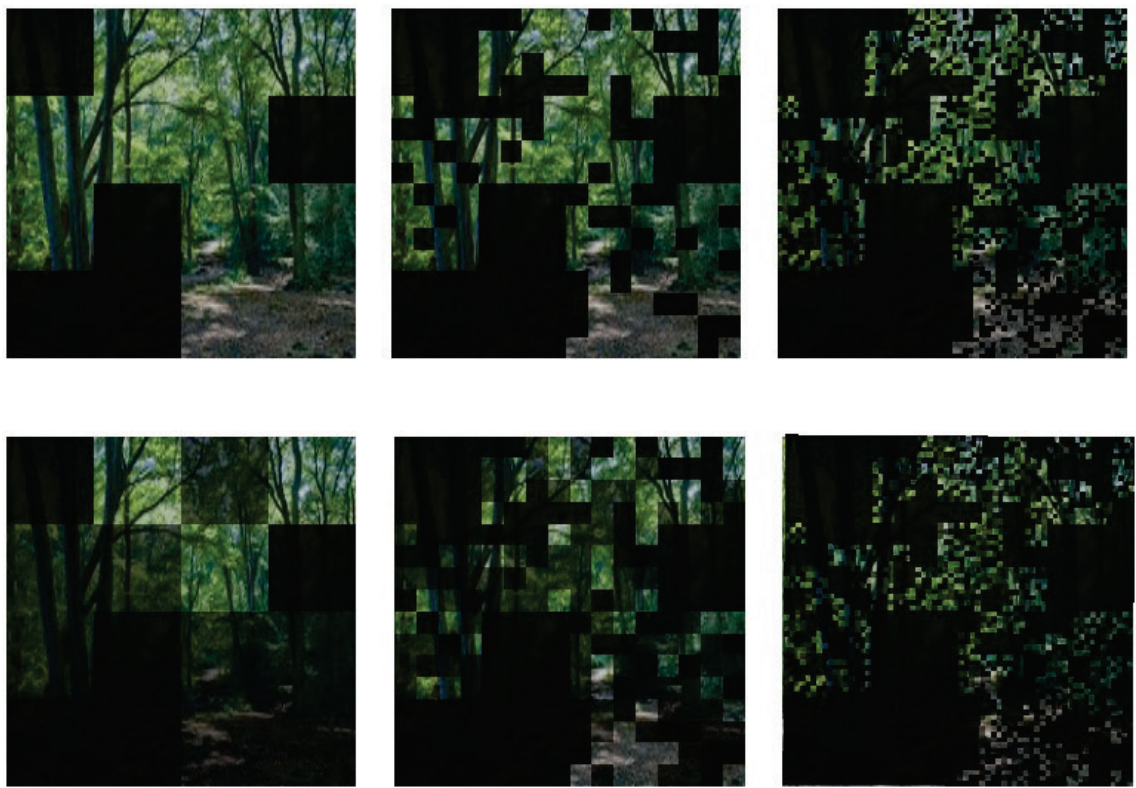

Figure 3 Façade glass surfaces with panels of different size and transparency level

The idea about a larger number of states of living cells within the random curds model in architectural and urban compositions is not new. One of the ways to increase the number of states [14] is to overlap several random curds mathematical models with different interpretations (contents) of living cells. The proposed procedure has shown its possibilities and its advantages, but also its limitations as a mathematical model of natural 
rhythm in architectural and urban compositions. The fact that during the overlap, we can have situations when one cell has two contents that cannot have the same interpretation, or cannot successfully adapt to each other, as well as the limitation of the proposed procedure in relation to the number of possible states - we cannot have any number as the number of possible states, but only 2 , $4,8,16$, etc. has initiated new ideas and approaches presented in this paper. This paper represents a continuation of research in that direction.

\section{MODIFICATIONS OF THE RANDOM CURDS MODEL}

Abandoning the idea of dividing all cells to new scaled cells with the scaled grid network from the previous step, not just the living cells, will not alter the random curds geometry, it will just enable a new interpretation of the model and its further modification.

In each subsequent step, every cell is divided to new scaled cells with the scaled grid network from the previous step. Each of these new cells inherits the attribute of its parent, the cell it originates from. It is living if it originates from a living cell, and dead if it originates from a dead cell. The probability of survival of each living cell in a new scale is $p$ and of non-survival is $1-p$. Each dead cell retains its state with a probability of 1. An event where a dead cell is brought back to life is a stochastically impossible event (with a probability 0 ), and cannot significantly affect the resulting random curds geometry.

This way, a partially modified random curds model is also observed as a Markov chain through several scales and with two states: "dead cell" (state 0) and "living cell" (state 1), with transition probabilities of $p_{00}=1, p_{01}=0$, $p_{10}=1-p, p_{11}=p$ (Fig. 4). The value of probability $\mathrm{p}$ is variable through iterations.

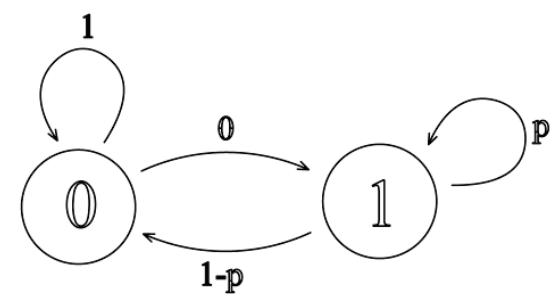

Figure 4 Random Curds as a Markov chain with two states

Observing the procedure of curdling as a Markov chain can be explained in another way. The crucial role in this approach belongs to the observation that both the living cells from that iteration and the dead cells from that step and all previous steps are present within a single iteration of a random curds fractal. If we consider all of them as cells within one iteration, the probability of survival of living cells is $\mathrm{p}$ and the probability of nonsurvival is $1-p$, while the dead cells will retain their state with probability 1 (Fig. 4). Compared to the existing curdling procedure that generates a random curds fractal, a deterministically certain event where dead cells do not alter their state has been replaced with a stochastically certain event realized with probability 1 . The probability that a dead cell will change its state is 0 , so the deviation from the random curds geometry is negligible, or practically does not exist.

Accepting a partially corrected curdling procedure, the number of state of living cells within the random curds model can be simply increased. We can speak about any finite, or countable infinite set of states. Fig. 5 shows a random curds model with two states of living cells, such as the one realized in the example of transparency (Fig. 3 - lower row of images). In this very simple example, all transition probabilities are equal: $p_{11}=p_{12}=p_{21}=p_{22}=$ 0.35 in the first and second iteration, and $p_{11}=p_{12}=p_{21}=$ $p_{22}=0.3$ in the third iteration.

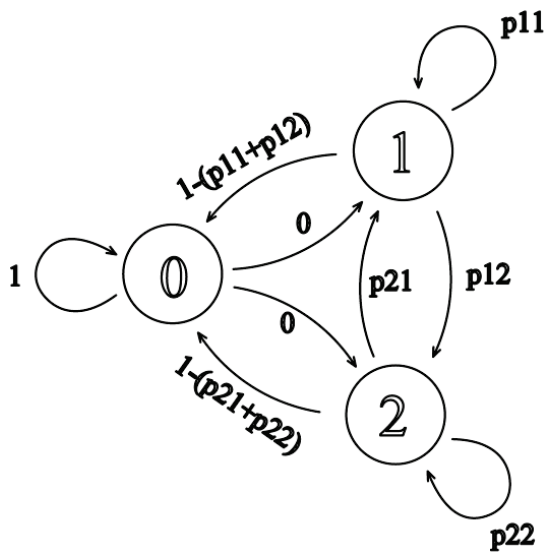

Figure 5 Markov chain with three states - random curds with two states of living cells

\subsection{Random curds with Multiple States as a Markov Chain}

A Markov chain is a sequence of random variables.

$X_{0}, X_{1}, X_{2}, \ldots$, where indexes $0,1,2 \ldots$ represent discrete moments (instants) or discrete values of another parameter expressed with whole numbers, such that from the standpoint of the present, the future is conditionally independent of the past, i.e.

$$
\begin{aligned}
& P\left\{X_{n}=i_{n} \mid X_{n-1}=i_{n-1}, \ldots, X_{1}=i_{1}, X_{0}=i_{0}\right\}= \\
& =P\left\{X_{n}=i_{n} \mid X_{n-1}=i_{n-1}\right\} .
\end{aligned}
$$

Thus, the distribution of states in the next step depends only on the current - present state and is determined by the transition probability matrix $\boldsymbol{M}=\left[p_{i j}\right]$, where $p_{i j}=P\left\{X_{n}=j \mid X_{n}=i\right\}$ of the probability passes from state $i$ to state $j$, which belong to a set of possible states. The number of possible states is finite, or countably infinite.

Example 1 In this example, we will describe a modified random curds model with multiple states, presented with the use of colors. The set of states is composed of whole numbers from 0 to 255 , with a defined cell color (shades of gray from black to white). The starting cell (zero iteration) is in one of the possible states. Each cell created by a division of the original cell inherits that state, survives in the first iteration, and reduces its value by 40 with probability $p_{1}$, survives and increases its value by 40 with probability $p_{2}$ and does not survive with probability $1-\left(p_{1}+p_{2}\right)$ (Fig. 6). Nonsurviving cells are marked as state 0 (black color). Addition and subtraction are defined as a clock system in which clock hands move from 0 to 255 : 


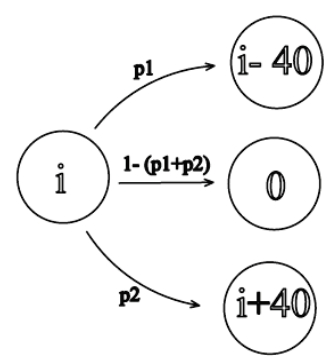

1 st iteration

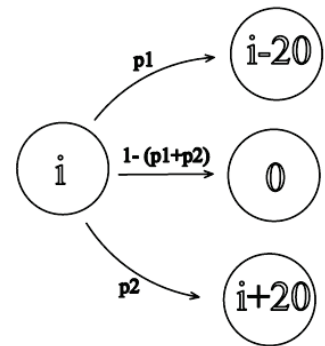

$2^{\text {nd }}$ iteration

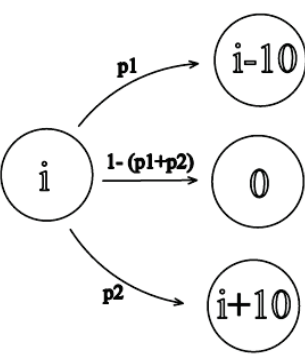

$3^{\text {rd }}$ iteration

Figure 6 Presentation of state changes within the model

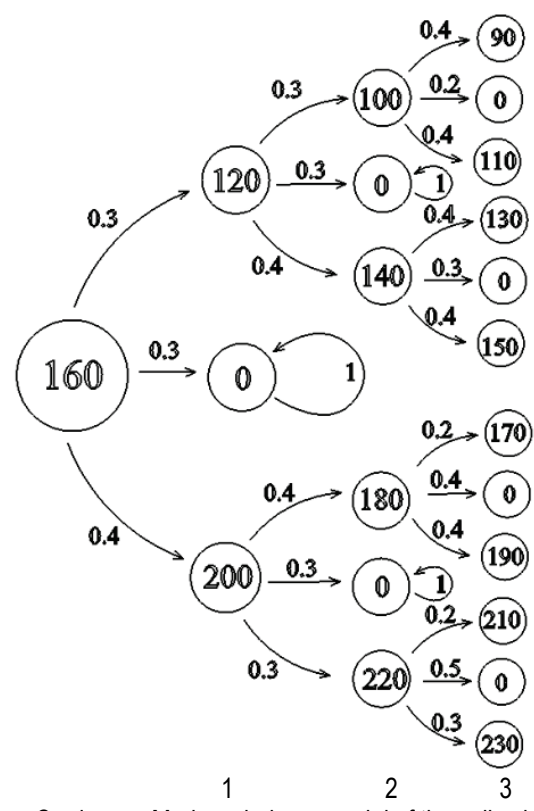

Figure 7 Random Curds as a Markov chain - a model of the cell colour change

$a+b=\left\{\begin{array}{l}a+b, a+b \leq 255 \\ a+b-255, a+b>255\end{array}\right.$

$a-b=\left\{\begin{array}{l}a-b, a-b \geq 0 \\ a-b+255, a-b<0\end{array}\right.$

In each subsequent iteration, the change of state is reduced. In the second iteration, states change by 20 , in the third by 10 , etc.

Transition probabilities $p_{1}$ and $p_{2}$ are variable, meaning they depend on the previous and subsequent state and change through iterations. The number of living cells increase. In the first iteration, we have two states of living cells, which, together with the dead cell, is three states in total; in the second iteration, we have four different states of living cells, and the dead cell as the fifth, etc. In the $n^{\text {th }}$ iteration, we would have $2^{n}$ states of living cells, or $2^{n}+1$ states in total. The number of possible states increases together with the total number of cells, which represents an additional benefit of the model.

A model concept, with initial color state 160, all states through three iterations and transition probabilities, is shown in Fig. 7. Three different realization variants of this model, with the same initial state and fixed transition probabilities are shown in Fig. 8. We showed the first three iterations for each variant.
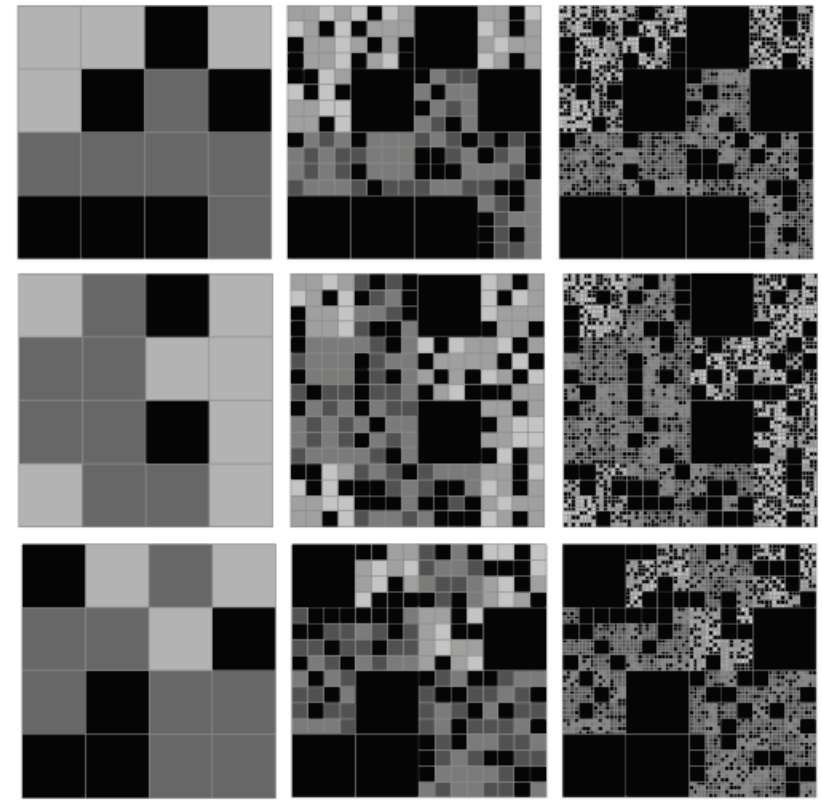

Figure 8 Random curds as a Markov chain - realizations of the color change model (3 iterations)

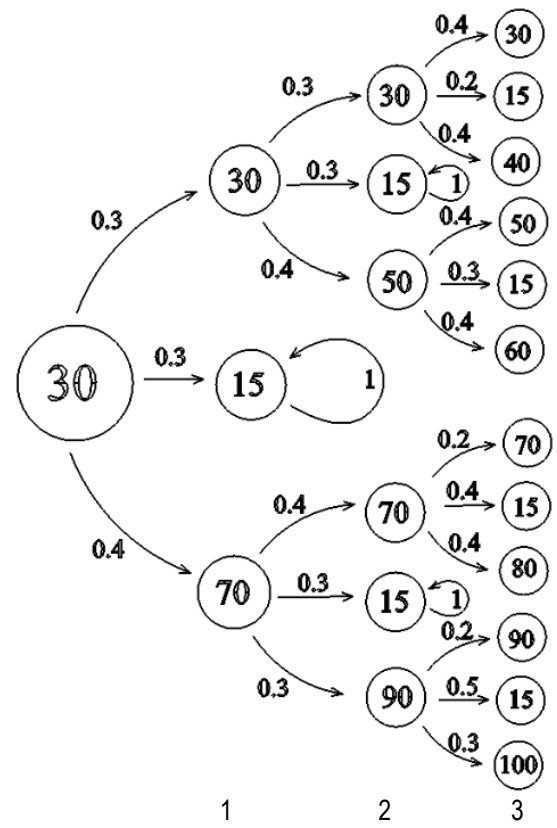

Figure 9 Random curds as a Markov chain - model of a transparent glass wall

Example 2 The subject of this example is a transparent glass wall. As a mathematical model, it is similar to the previous example, which refers to different shades of gray. The set of states consists of whole numbers from 15 to 100 , which define a cell in percentages. The initial cell (zero iteration) is in one of 
the possible states. Each cell created by a division of the initial cell inherits that state, survives in the first iteration, and retains that state with probability $p_{1}$, survives and increases its transparency for $40 \%$ with probability $p_{2}$, and does not survive with probability $1-\left(p_{1}+p_{2}\right)$ (Fig. 9). Non-surviving cells are marked as state 15 (transparency $15 \%$ ). Addition and subtraction are defined as a clock system where clock hands move from 15 to 100. In each subsequent iteration, the change of state is reduced. In the second iteration, states change by 20 , in the third by 10 , etc. Transition probabilities can be random, but we took them from the previous model for detailed research of forms that appear there.
A model concept with the initial state of transparency of $30 \%$, all states through three iterations and transition probabilities, is shown in Fig. 9. Several variants of the third iteration of this model, with identical initial state and fixed transition probabilities are shown in Fig. 10.

Viewing transition values as parametric values and implementing genetic algorithms, we attempted to find variants with preselected values of average transparency, regarding the issue of the necessary amount of natural light. Fig. 11 shows interesting obtained solutions for average transparencies of $30 \%, 40 \%$ and $50 \%$, respectively.
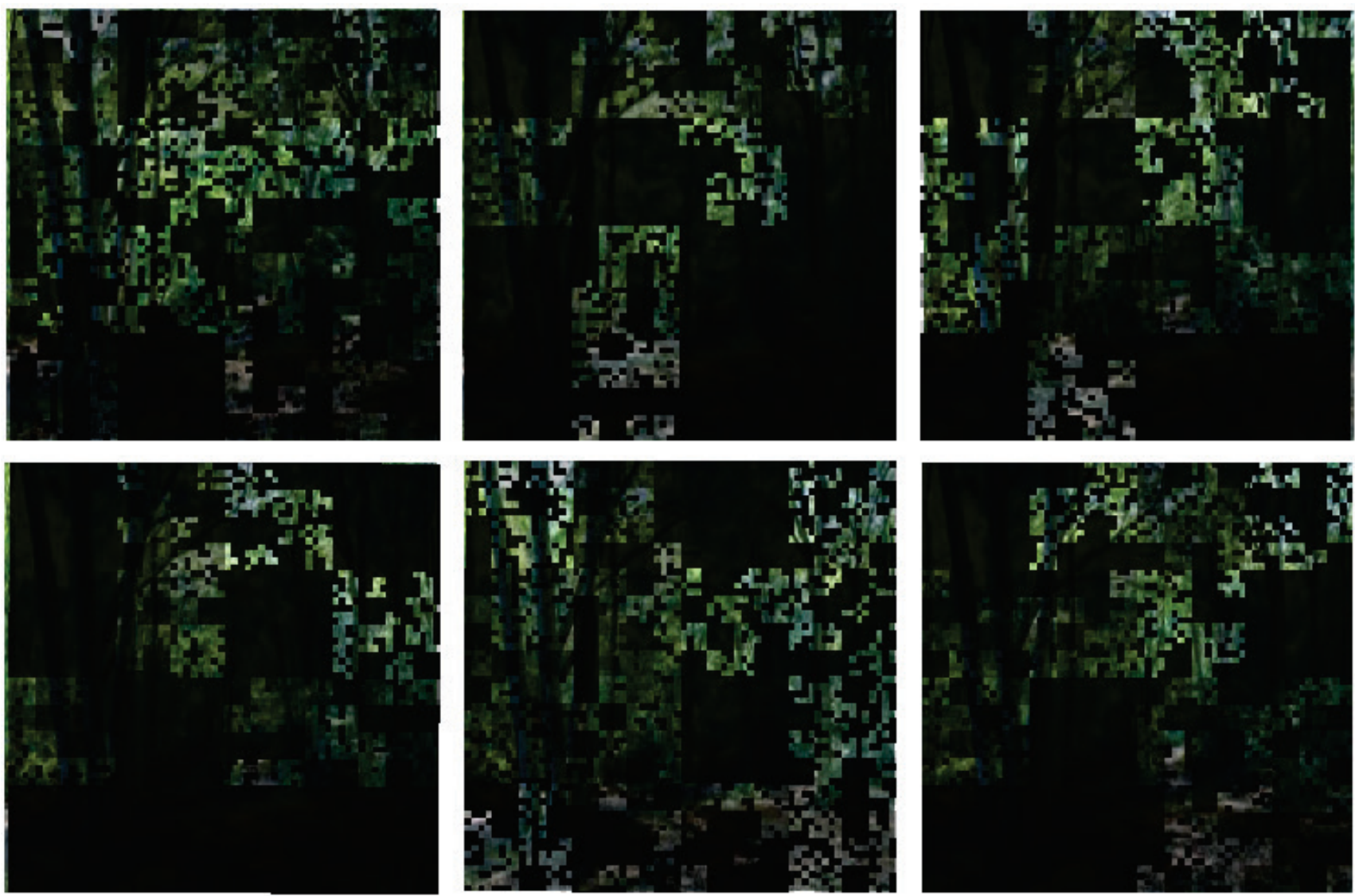

Figure 10 Random curds transparent glass wall
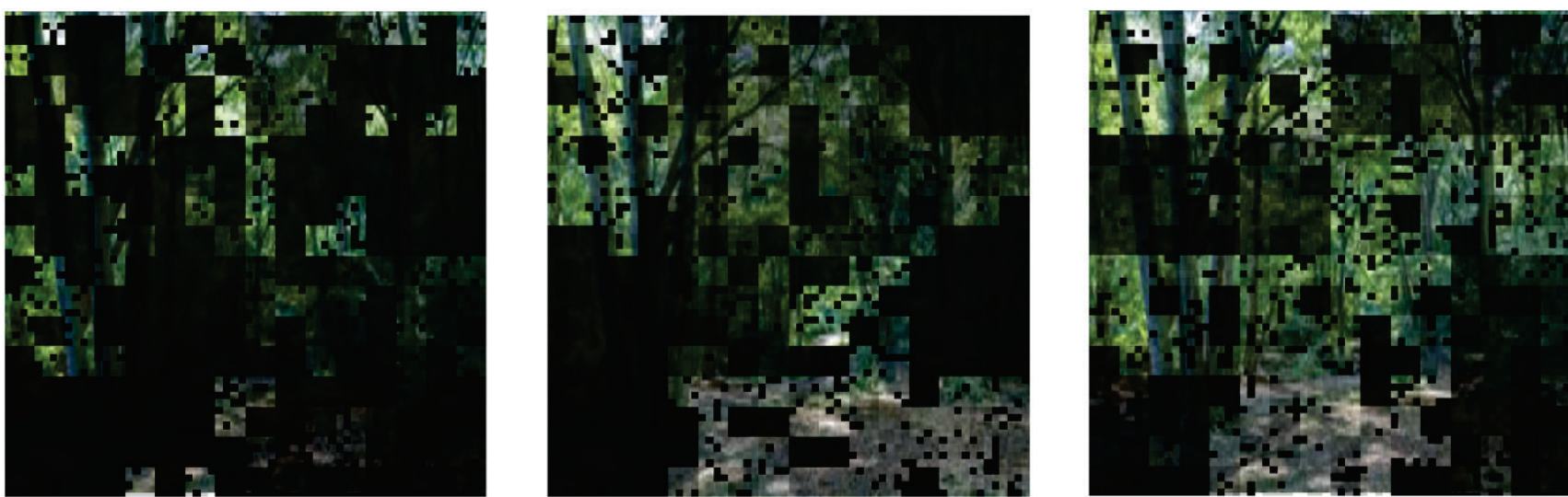

Figure 11Random curds transparent glass wall with different levels of average transparency

Example 3 In this example, we will use a random curds model with multiple states (Fig. 12) to generate fractal rhythm in an urban composition for outdoor activities - a small park.
Initial state 1 indicates the parcel intended for the park. In the first iteration, that area is divided into cells. State 11 in that iteration indicates the area in the shade, and state 12 indicates sunlit surfaces, from the standpoint 
of space used for spending time in the sun. In the second iteration, within 11 , state 111 are positions of the trees that cast shade, and state 112 are positions of the benches in the shaded area. Within 12, state 121 indicates free surfaces covered with grass, and 122 positions of the benches in the sunlit part. State 0 in all iterations represents dead cells, and they are paved surfaces in the model. We choose transition probabilities in accordance with our needs, for example, depending on the climate. With a finite selection of probabilities, each stochastic model offers a number of solutions. Three possible solutions for this model, with selected probabilities shown in Fig. 12, are schematically represented in Fig. 13.

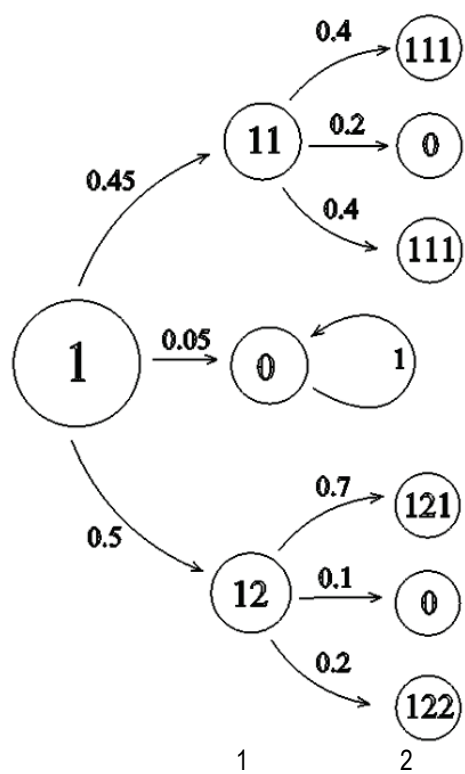

Figure 12 Random curds as a Markov chain - model of a park
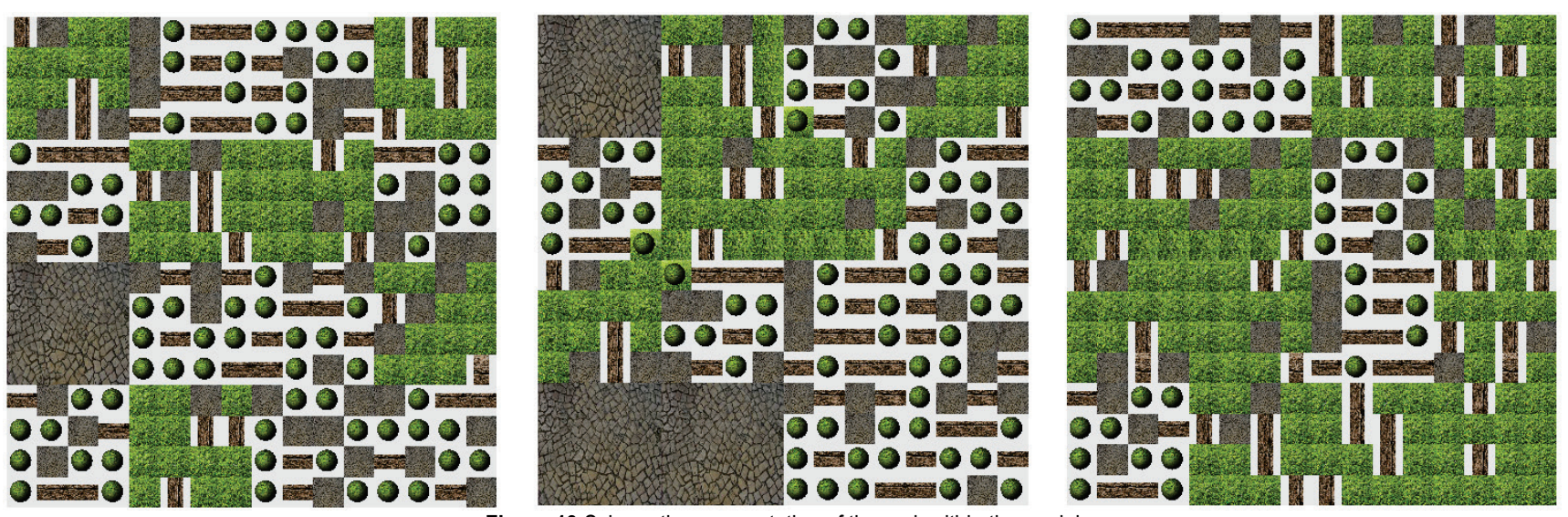

Figure 13 Schematic representation of the park within the model

\section{CONCLUSION}

Selected probabilities as parametric values within the model represent our choice in terms of desired expected values. However, we will not always obtain solely expected mean values. Random access implies deviations from those values. In the last example of the park, in the first iteration, the total probability of survival is 0.95 , and the probability of a cell's non-survival is 0.05 . This illustrates the need for a small number of dead cells, although this number is not quite determined. The number of dead cells in the first iteration has binomial distribution and the probability that exactly $k$ cells are dead is: $P\{X=k\}=\left(\begin{array}{l}n \\ k\end{array}\right) q^{k}(1-q)^{n-k}$ where $n$ is the total number of cells, and $q$ is the probability that a cell will not survive. The expected mean value is $E=n q$. In our case, $n$ $=16(4 \times 4$ grid $)$, so the expected number of dead cells is $E$ $=0.05 \cdot 16=0.8 \approx 1$. The probability that there will be no dead cells is 0.440 , and the probabilities that there will be one, two, three and four dead cells are $0.371,0.146,0.036$ and 0.006 respectively. Probabilities of occurrence of other values are very small, in $10^{-4}$ order of magnitude, and higher, 0.001 in total. Variants in Fig. 13 have one, two, three and no dead cells, in that order. By increasing the probabilities, we increase the expected value, and therefore the real number of dead cells, which gives us a certain ability to manage the model, but does not lead to final solution with completely predefined characteristics. Similarly, one can discuss and analyze distribution of living cells in one of two possible states of the first iteration for example, the number of living cells in one of four possible states of the second iteration, i.e. distribution of all random variables within the model, which is not the objective of this paper. We simply made an illustration that contributes to a better understanding and analysis of the model's possibilities.

Therefore, by selecting probabilities as parametric values of the model, we narrow the scope of potential outcomes and navigate the model toward a final solution, but we are still left with a number of possible variants at our disposal. In deterministic parametric models, the selection of a variant by the architect represents a significant advantage, but in the case of stochastic models, it is hard to inspect all possible variants and find a reason to accept one of them. What remains is the possibility of selecting the final variant using genetic algorithms which are based on a criterion (known as fitness function), like we could select variants with average transparency of $30 \%, 40 \%$ and $50 \%$ in the example of the transparent glass wall in Example 2. In the case of the park, we could incorporate a paved surface, 
number of tress, lawn area, number of benches, etc. in the fitness function. We could search for a variant with minimum, maximum, or any preselected value for each of the selected functions. Genetic algorithms provide a sufficient number of alternative solutions as the result of optimization, so the architect almost always has the possibility of final selection. Finally, we will add a closing comment in support of the modified random curds model. Redistribution of "survival" probabilities between multiple states of living cells does not damage the visual effect of random clustering similar to that observed in nature. The basic idea behind the random curds model that not all cells "survive", is incorporated in transition probabilities of a Markov chain with multiple states, and it provides that fractal random curds geometry. A fractal random curds structure, as a whole, represents a unity of all its disjunctive parts that originate from different states, and additionally, each of those parts can be observed as a random curds fractal in a certain context.

\section{REFERENCES}

[1] Arnheim, R. (1974). Art and visual perception: A psychology of the creative eye. University of California, Berkeley and Los Angeles Press.

[2] Ching, D. K. F. (2007). Architecture: Form, space \& order. New York: International Thomson Publishing.

[3] Rasmussen, S. E. (1959). Experiencing Architecture. MIT Press.

[4] Bovill, C. (1996). Fractal geometry in architecture and design. Boston: Birkhäuser. https://doi.org/10.1007/978-1-4612-0843-3

[5] Bovill, C. (2000). Fractal geometry as design aid. Journal for Geometry and Graphics, 4(1), 71-78.

[6] Mandelbrot, B. B. (1982). The fractal geometry of nature. New York: Freeman.

[7] Kappraff, J. (2002). Beyond Measure: A Guided Tour Through Nature, Myth, and Number. World Scientific Publishing Co. https://doi.org/10.1142/4767

[8] Alexander, C. (1964). Notes on the Synthesis of Form. Harvard University Press.

[9] Stiny, G. (2006). Shape: Talking about Seeing and Doing. Massachusetts: The MIT Press.

[10] Evans, R. (1995). The projective cast: Architecture and its three geometries. Cambridge, Massachusetts: The MIT Press.

[11] Ostwald, M. J. (2003). Symbols of evolution, signs of regression: Mies and the politics of geometry. // In M. Gusheh \& N. Stead (Eds.). The Proceedings of the $X X^{\text {th }}$ Annual Conference of the Society of Architectural Historians, Australia and New Zealand, Sydney: Society of Architectural Historians, Australia and New Zealand, 226231.

[12] Ostwald, M. (2009). Fractal Architecture: Knowledge Formation Within and Between Architecture and the Sciences of Complexity. Germany: Verlag Dr. Muller.

[13] Ostwald, M. J. (2000). Under siege: The golden mean in architecture. Nexus Network Journal, 2, 75-81. https://doi.org/10.1007/s00004-999-0010-z

[14] Ostwald, M. J. (2001). Fractal architecture: Late twentieth century connections between architrcture and fractal geometry. Nexus Network Journal, 3(1), 73-83. https://doi.org/10.1007/s00004-000-0006-1

[15] Prigogine, I. (1997). The End of Certainty: Time, Chaos and the New Laws of Nature. New York: The Free Pres.

[16] Jevrić, M., Knežević, M., Kalezić, J., Kopitović-Vuković, N. \& Ćipranić, I. (2014). Application of Fractal Geometry in Urban Pattern Design. Tehnicki vjesnik-Technical Gazette, 21(4), 873-879.

[17] Ćirović, I. (2014). Random curds as mathematical models of fractal rhythm in architecture. SPATIUM International Review, 31, 79-84. https://doi.org/10.2298/SPAT1431079C

[18] Devetaković, M., Petruševski, I., Ćirović, I. \& Petruševski, Lj. (2015). Fractal parametric models of urban spaces. Tehnicki vjesnik-Technical Gazette, 22(6), 1547-1552. https://doi.org/10.17559/TV-20150121222048

[19] Voss, F. R. (1988). Fractals in nature: From characterization to simulation. In Peitgen, H. O. and Saupe, D. (eds.) / The science of fractal images. New York : Springer-Verlag, 21-70. https://doi.org/10.1007/978-1-4612-3784-6_1

[20] Peitgen, H. O., Jurgens, H. \& Saupe, D. (2004). Chaos and fractals: New frontiers of science. New York : SpringerVerlag. https://doi.org/10.1007/b97624

[21] Taylor, R. \& Sprott, J. C. (2008). Biophilic fractals and the visual journey of organic screen-savers. Nonlinear Dynamics, Psychology, and Life Sciences, 12(1), 117-129.

[22] Richards, R. (2001). A new aesthetic for envirinmental awareness: Chaos theory, the beauty of nature, and our broader humanistic identity. Journal of Humanistic Psychology, 41(2), 59-95. https://doi.org/10.1177/0022167801412006

[23] Petrusevski, Lj., Devetaković, M. \& Mitrović, B. (2009). Self-replicating systems in spatial form generation: The concept of cellular automata. SPATIUM International Review,19, 8-14. https://doi.org/10.2298/SPAT0919008P

\section{Contact information:}

Ljiljana PETRUŠEVSKI, PhD, Professor

University of Belgrade, Faculty of Architecture

Bulevar kralja Aleksandra 73/2, 11000 Belgrade, Serbia

E-mail: ljpetrusevski@gmail.com

\section{Ivana ĆIROVIĆ, PhD, Senior Lecturer}

Business Technical College of Vocational Studies

Trg Svetog Save 34, 31000 Užice, Serbia

E-mail: ivana.cirovic1@gmail.com 